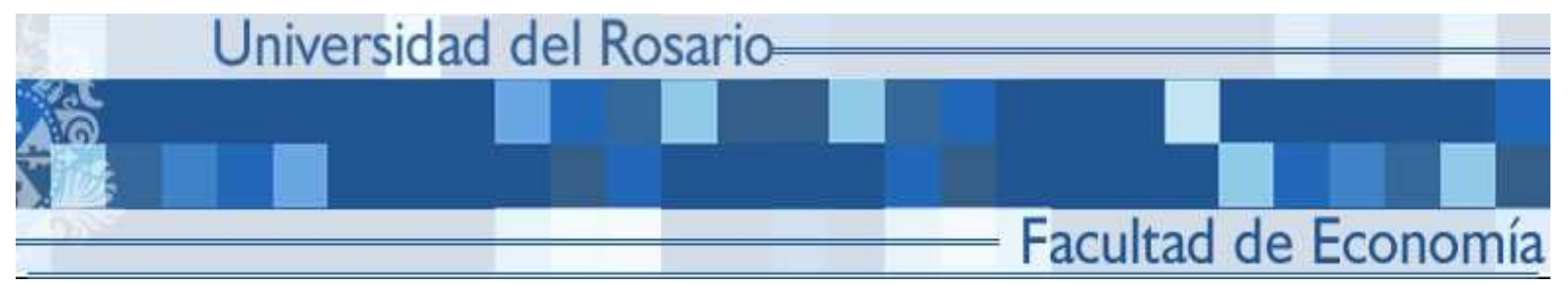

\title{
ALLOCATION RULES ON NETWORKS
}

Rahmi Ilkiliç

Çâğatay Kayi

\section{SERIE DOCUMENTOS DE TRABAJO}

No. 118

Marzo 2012 


\title{
Allocation Rules on Networks*
}

\author{
Rahmi İlkılıç and Çağatay Kayı ${ }^{\ddagger}$
}

\begin{abstract}
When allocating a resource, geographical and infrastructural constraints have to be taken into account. We study the problem of distributing a resource through a network from sources endowed with the resource to citizens with claims. A link between a source and an agent depicts the possibility of a transfer from the source to the agent. Given the supplies at each source, the claims of citizens, and the network, the question is how to allocate the available resources among the citizens.

We consider a simple allocation problem that is free of network constraints, where the total amount can be freely distributed. The simple allocation problem is a claims problem where the total amount of claims is greater than what is available. We focus on consistent and resource monotonic rules in claims problems that satisfy equal treatment of equals. We call these rules fairness principles and we extend fairness principles to allocation rules on networks. We require that
\end{abstract}

${ }^{*}$ We would like to thank Paula Jaramillo, Herve Moulin, and William Thomson for detailed comments on an earlier draft of the paper. We also thank the seminar participants at First Caribbean Game Theory Conference, Katholieke Universiteit Leuven, University of Tsukuba, Maastricht University, Universidad del Rosario, and Hausdorff Research Institute for Mathematics for valuable discussions. Part of the research was completed when R. İlkılıç and Ç. Kayı were affiliated with Maastricht University.

${ }^{\dagger}$ Department of Economics, Bilkent University. Email: rahmi.ilkilic@bilkent.edu.tr. R. İlkılıç acknowledges the support of the European Community via Marie Curie Grant PIEF-GA-2008-220181.

${ }^{\ddagger}$ Faculty of Economics, Universidad del Rosario. Email: kayi.cagatay@urosario.edu.co. Ç. Kayı thanks the Netherlands Organization for Scientific Research (NWO) for its support under grant VIDI-452-06-013 and gratefully acknowledges the hospitality of the Hausdorff Research Institute for Mathematics for inviting as a visiting fellow to Trimester Program on Mechanism Design and Related Topics in 2009. 
for each pair of citizens in the network, the extension is robust with respect to the fairness principle. We call this condition pairwise robustness with respect to the fairness principle. We provide an algorithm and show that each fairness principle has a unique extension which is pairwise robust with respect to the fairness principle. We give applications of the algorithm for three fairness principles: egalitarianism, proportionality and equal sacrifice.

Keywords: Networks, Claims Problems, Egalitarianism, Proportionality, Equal Sacrifice.

JEL-Numbers: D61, D85, Q20.

\section{Introduction}

The world has become a densely connected network, especially for markets and natural resources. Given geographical or infrastructural constraints, it is important to understand how scarce resources should be allocated. An example where such network constraints are essential is fresh water resources. As a result of increasing population and developing economies, there is a growing need for water. The principal problem is to have an efficient and fair allocation of resources Ansink and Weikard, 2009: Hoekstra, 2006). Some other examples are aid relief during disaster situations (Özdamar et al. 2004), common property fisheries (İlkılıç, 2007), and the distribution of utilities like electricity and natural gas when there is a supply shock.

We study the problem of distributing a resource through a bipartite network between citizens with positive claims, needs, or entitlements and sources that are endowed with a limited amount of the desired resource. If there is a link between a source and a citizen, then the citizen can receive the resource from the source. Each source has a limited supply of the resource and each citizen has a claim on the resource. Given the network constraints, the demand of citizens, and the supplies at each source, the question is how to allocate the resource among the citizens. An allocation rule assigns to each citizen a quantity of resource satisfying the following feasibility constraints: First, a citizen can not receive more than his demand. Second, a source can not deliver more than its supply.

We study those problems where total demand exceeds total supply where all the agents suffer from the scarcity 1 When individuals have claims on a resource

\footnotetext{
${ }^{1}$ If there is a group of agents on the network whose claims can be completely satisfied
} 
that sum up to more than what is available, how should the resource be divided? This problem is a claims problem, formally introduced by O'Neill (1982). Several rules are commonly used in practice and analyzed in theoretical work (Thomson, 2003, 2006).

An allocation problem is defined by the supplies at the sources, the claims of citizens, and the network. The question is how to allocate the resources among the citizens. First, we define a simple allocation problem that is free of network constraints, where the total amount can be freely distributed between the agents. The simple allocation problem is in fact a claims problem. We focus on a subset of rules in claims problems. A rule satisfies equal treatment of equals if two citizens with equal claims receive equal amounts. A rule is resource monotonic if when the resource increases, each citizen receives at least as much as he did initially. For the next property, suppose a rule has been applied to a claims problem and some citizens leave with what they are prescribed by the rule. If we apply the rule to the problem with the remaining citizens and the remaining resources, then the initial prescribed allocation should not change for the remaining ones. A rule is consistent if it satisfies this invariance property.

We refer to the rules that are consistent and resource monotonic satisfying equal treatment of equals as fairness principles. We extend fairness principles to allocation rules on networks. We require that for each pair of citizens in the network, the extension is robust with respect to the fairness principle. We call this condition pairwise robustness with respect to the fairness principle. We provide an algorithm to extend a fairness principle to an allocation rule which is pairwise robust with respect to the fairness principle (Theorem 1). This algorithm is parallel to the ascending algorithms used in Moulin (1999) and Bochet et al. (2010, 2011). Then, we show that each fairness principle has a unique extension which is pairwise robust with respect to the fairness principle (Theorem 2 ).

The literature on flow sharing on networks has focused on computation of egalitarian solutions (Megiddo, 1974, 1977, Brown, 1979; Hall and Vohra, 1993). Several allocation rules for allocation problems on networks have recently been introduced and axiomatized in Branzei et al. (2008), Bjørndal and Jörnsten (2010), Bochet et al. (2010, 2011), and Moulin and Sethuraman (2011).

Branzei et al. (2008) represent a claims problem as a standard flow problem on a simple network and implement some known rules via suitable cost functions in the related minimum cost flow problem. Bjørndal and Jörnsten (2010) provide without any burden on others, we can simply take those agents put of the network and focus on the "genuine" problem 
an extension of two simple allocation rules (the contested-garment rule and the constrained equal awards rule) by applying to each two person problem.

Bochet et al. (2010) study the egalitarian rule for allocation problems on networks, which they characterize with Pareto optimality, equal treatment of equals and strategy-proofness. Their egalitarian rule is an extension of the uniform rule (Sprumont, 1991) for the simple allocation problem to an allocation problem on networks in an agent consistent way, meaning if an agent leaves the problem with her share and the corresponding amounts are reduced from the sources she received her share, then in the remaining network the agents should receive the same amounts as in the original problem. Our aim in this paper is to extend all consistent and resource monotonic rules satisfying equal treatment of equals for the simple problem to a network allocation problem in an agent consistent manner. Our pairwise robustness captures this consistency requirement.

Moulin and Sethuraman (2011) provides an alternative extension of simple allocation rules in a source consistent fashion, meaning if a source leaves the problem with its resources and the corresponding amounts are reduced from the agents receiving them, then the new problem should allocate the agents the shares they received in the original problem plus the amounts allocated from the deleted source. Bochet et al. (2011) studies the problem of balancing the demands and supplies between agents where links depict the transfer from a supplier to a demander. That is different from a classic allocation problem as the agents receive the commodity they desire from other agents (Klaus et al., 1997, 1998).

The allocation problem we study is different from models where agents are located sequentially on a line or the so-called river sharing (Ambec and Sprumont, 2002: Ambec and Ehlers, 2008, Ansink and Weikard, 2011). A river sharing problem can be written as an allocation problem on a network where agents' access to sources are hierarchical ${ }^{2}$ Hence our model is more general than a river sharing problem as we have no restrictions on the possible networks between sources and agents. Our model is also different than the division of a single commodity supplied by multiple sources as studied in Kar and Kibris (2008). There, although an agent, a priori, can consume from any source, she must receive all her endowment from a single

\footnotetext{
${ }^{2}$ In more detail, the river sharing can be written as an allocation problem on a network in the following manner. The initial stream reaching the first agent on the river and the rainfall received by every agent are the sources in our network. The last agent on the river has access to all sources. The second from the last agent has access to all sources except the rainfall of the last agent and in general an agent has access to all sources except the rainfall of her downstream agents.
} 
source whereas an agent in our model can receive her share from several sources which she has access to.

In Section 2, we introduce the model and some properties of fairness principles. In Section 3, we present the algorithm and give the results. In Section 4 , we give three applications of the algorithm. We conclude in Section 5 .

\section{Model}

Let $\mathbf{S}$ be the set of sources and $|S|=m$, and $\mathbf{C}$ be the set of citizens and $|C|=n$. Each source $\mathbf{t} \in S$ has a non-negative supply $\mathbf{s}_{\mathbf{t}} \in \mathbb{R}_{+}$and each citizen $\mathbf{i} \in C$ has a non-negative claim $\mathbf{c}_{\mathbf{i}} \in \mathbb{R}_{+}$for the resource.

Let $s=\left(s_{1}, s_{2}, \ldots, s_{m}\right)$ be the supply vector and $c=\left(c_{1}, c_{2}, \ldots, c_{n}\right)$ be the claims vector. The sources and citizens are embedded in a network in which citizens can acquire the resource only from the sources they are connected to. A bipartite graph $\mathrm{g} \subseteq S \times C$ consists of links between nodes in $S$ and $C$. If a link connects a source $t$ to a citizen $i$ in $g$, i.e., $t i \in g$, then it is possible for citizen $i$ to acquire the resource from source $t$. We assume that $g$ is connected. If it is not, then we can treat each connected component of $g$ as a separate problem. Let $\mathcal{G}_{n \times m}$ be the set of all connected bipartite graphs between $S$ and $C$.

A subgraph of $g$ is a graph $\mathbf{g}(\mathbf{T}, \mathbf{D}) \subseteq g$ such that $T \subseteq S, D \subseteq C$ and each link in $g$ that connects a source in $T$ to a citizen in $D$ is a member of $g(T, D)$, i.e., $g(T, D)=g \cap(T \times D)$. For a subgraph $g(T, D)$ of $g$, we denote by $g(S \backslash T, C \backslash D)$, the subgraph of $g$ that results when we remove all nodes in $T \cup D$ from $g$. Let $\mathbf{N}_{\mathbf{g}}(\mathbf{T})$ be the set of citizens connected to the set of sources $T$ in $g ; N_{g}(T)=$ $\{i \in C$ such that $t i \in g$ for some $t \in T\}$. Similarly, $\mathbf{N}_{\mathbf{g}}(\mathbf{D})$ be the set of sources connected to the set of citizen $D$ in $g ; N_{g}(D)=\{t \in S$ such that $t i \in g$ for some $i \in$ $D\}$.

An allocation is a vector $\mathbf{q}=\left(q_{1}, q_{2}, \ldots, q_{n}\right) \in \mathbb{R}_{+}^{n}$ showing how much resource is allocated to each citizen. A transfer of resources, or simply a flow, is a vector $\phi$ $\in \mathbb{R}_{+}^{S \times D}$, where $\phi_{t i}$ is the amount sent from source $t$ to citizen $i$ such that if $t i \notin g$, then $\phi_{t i}=0$.

An allocation $q$ is feasible if there is a flow $\phi \in \mathbb{R}_{+}^{S \times D}$ that supports it, i.e., for each citizen $i \in C, q_{i}=\sum_{t \in N_{g}(i)} \phi_{t i}$ and for each source $t \in S, \sum_{i \in N_{g}(t)} \phi_{t i} \leq s_{t}$. An allocation $q$ is efficient if it is feasible and there is no other feasible allocation $q^{\prime}$ such that for each citizen $i \in C$, we have $c_{i} \geq q_{i}^{\prime} \geq q_{i}$ and $\sum_{i} q_{i}^{\prime}>\sum_{i} q_{i}$. A feasible allocation $q$ satisfies claim boundedness if for each citizen $i \in C, q_{i} \leq c_{i}$. 
An allocation problem is a triple $\boldsymbol{R}=(s, c, g)$ such that

$$
\text { for all } T \subset S, \sum_{t \in T} s_{t}<\sum_{i \in N_{g}(T)} c_{i}
$$

Note that no subset of sources has enough resource to satisfy the claims of the citizens connected to them. Hence, the allocation problem is "genuine" in the sense that a citizen receives the resource always at the expense of some other citizen. Let $\mathcal{R}=\mathbb{R}_{+}^{m} \times \mathbb{R}_{+}^{n} \times \mathcal{G}_{m \times n}$ be the set of allocation problems. See Figure 1 for an example of an allocation problem.

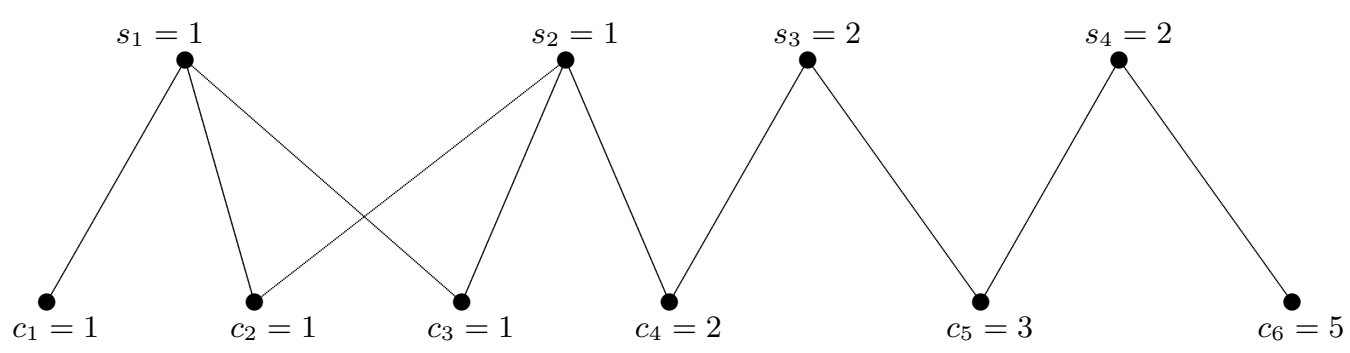

Figure 1: An example of an allocation problem: $R=(s, c, g)$ is an allocation problem with $S=\{1,2,3,4\}, C=\{1,2,3,4,5,6\}, s=(1,1,2,2)$, and $c=$ $(1,1,1,2,3,5)$.

An allocation rule $\varphi$ is a function which assigns to each allocation problem $(s, c, g)$ an efficient allocation that satisfies claim boundedness. Since each rule assigns an allocation to each problem, there is a flow supporting that allocation. If $\phi(s, c, g)$ is a flow that supports $\varphi(s, c, g)$, then for each citizen $i, \varphi_{i}(s, c, g)=$ $\sum_{t \in \mathcal{S}_{g}(i)} \phi_{t i}(s, c, g)$.

A simple allocation problem is a pair $\boldsymbol{P}=(\boldsymbol{c}, \boldsymbol{\omega})$ such that $\sum_{i \in C} c_{i} \geq \omega$. Note that $P$ represents the problem of allocating an amount $\omega \geq 0$ among the citizens in $C$. There is no restriction on the possible flows and $\omega$ can be distributed freely to citizens. Let $\mathcal{P}=\mathbb{R}_{+}^{m} \times \mathbb{R}_{+}$be the set of simple allocation problems.

A rule $\mathbf{f}$ is a function which assigns to each simple allocation problem $(c, \omega)$ an efficient allocation, i.e., $\sum_{i \in C} f_{i}(c, \omega)=\omega$ that satisfies claim boundedness, i.e., for each $i \in C, f_{i}(c, \omega) \leq c_{i}$. 
A rule $f$ satisfies equal treatment of equals if for each simple allocation problem $(c, \omega)$ and each pair of citizens $i, j \in C$ with $c_{i}=c_{j}$, we have $f_{i}(c, \omega)=$ $f_{j}(c, \omega)$, i.e. two citizens with equal claims receive equal amounts.

For the next property, suppose a rule has been applied to a claims problem and some citizens leave with what they are prescribed by the rule. If we apply the rule to the problem with the remaining citizens and the remaining resource, then the initial prescribed allocation does not change for the remaining ones. A rule is consistent if it satisfies this invariance property. Formally, a rule $f$ is consistent if for each simple allocation problem $(c, \omega)$, each $D \subset C$, and each $i \in C \backslash D$,

$$
f_{i}\left(c_{-D}, \omega-\sum_{j \in D} f_{j}(c, \omega)\right)=f_{i}(c, \omega)
$$

where $c_{-D}$ is the claims vector of the citizens in $C \backslash D$.

A rule is resource monotonic if when the resource increases, each citizen receives at least as much as he did initially. A rule $f$ is resource monotonic if for each pair of simple allocation problems $(c, \omega)$ and $\left(c, \omega^{\prime}\right)$ with $\omega^{\prime}>\omega$ and each $i \in C$, we have $f_{i}\left(c, \omega^{\prime}\right) \geq f_{i}(c, \omega)$.

We focus on consistent and resource monotonic rules in claims problems that satisfy equal treatment of equals. We call these rules fairness principles. Our aim is to extend each fairness principle to an allocation rule on a network. We require the extension to conserve the essence of the fairness principle. We formalize this requirement by the following definition:

Pairwise $\boldsymbol{f}$-Robustness: Given an allocation problem $(s, c, g)$ and a fairness principle $f$, an allocation $q$ is pairwise $f$-robust if for each pair of citizens $i, j \in$ $C$ with $f\left(\left(c_{i}, c_{j}\right), q_{i}+q_{j}\right)=\left(q_{i}^{*}, q_{j}^{*}\right)$, there exists no feasible allocation $q^{\prime}$ for the allocation problem $(s, c, g)$ such that for each $k \neq i, j, q_{k}^{\prime}=q_{k}$ and

$$
\left|q_{i}^{*}-q_{i}^{\prime}\right|<\left|q_{i}^{*}-q_{i}\right|
$$

An allocation rule $\varphi$ is pairwise $f$-robust if for each allocation problem $(s, c, g) \in$ $\mathcal{R}, \varphi(s, c, g)=q$ satisfies pairwise $f$-robustness.

Next, we construct an algorithm to extend any fairness principle $f$ to an allocation rule $\varphi^{f}$ that is pairwise $f$-robust. Then, we show that for each fairness principle $f$, there exists a unique allocation rule which is pairwise $f$-robust. 


\section{The Ascending Algorithm}

Let $f$ be a fairness principle. Given the claims of the citizens $c$, consider the simple allocation problem with a single source of capacity $\omega \geq 0$. For each citizen $i$, let $f_{i}(c, \omega)$ be the amount that citizen $i \in C$ would have received under the fairness principle $f$ in the simple allocation problem $(c, \omega)$. Note that by resource monotonicity, $f_{i}(c, \omega)$ is an increasing function of $\omega$.

We obtain the allocation rule $\varphi^{f}(s, c, g)$ by an ascending algorithm based on the following system $K(\omega)$ of inequalities where $\omega$ is a non-negative parameter:

$$
\sum_{i \in D} f_{i}(c, \omega) \leq \sum_{t \in N_{g}(D)} s_{t} \text { for all } D \subseteq C
$$

For $\omega=0$, equation (1) is satisfied for each $D \subseteq C$. For $\omega=\sum_{i \in C} c_{i}$, there exists $D \subseteq C$ such that

$$
\sum_{i \in D} f_{i}(\omega, c)>\sum_{t \in N_{g}(D)} s_{t}
$$

by construction. Hence, there exists a largest $\omega^{1}$ such that

$$
\begin{aligned}
\sum_{c_{i} \in D} q_{i}\left(c, \omega^{1}\right) & \leq \sum_{s_{t} \in N_{g}(D)} s_{t} \text { for each } D \subseteq C \\
\sum_{c_{i} \in D} q_{i}\left(c, \omega^{1}\right) & =\sum_{s_{t} \in N_{g}(D)} s_{t} \text { for some } D \subseteq C
\end{aligned}
$$

As $\sum_{t \in N_{g}(D)} s_{t}$ is a submodular function of $D$, there exists a unique largest $D^{1}$ such that equation 2 holds. The allocation $\varphi^{f}(s, c, g)=q$ is obtained by setting

$$
q_{i}=f_{i}\left(c, \omega^{1}\right) \text { for each } i \in D^{1}
$$

and assigning other agents their allocation in the reduced problem $\left(s \backslash N_{g}\left(D^{1}\right), c \backslash\right.$ $\left.D^{1}, g \backslash\left(N_{g}\left(D^{1}\right) \cup D^{1}\right)\right)$. That is, we look for the largest $\omega^{2}>0$ such that

$$
\begin{aligned}
\sum_{i \in D} f_{i}\left(c, \omega^{2}\right) & \leq \sum_{t \in N_{g}(D) \backslash N_{g}\left(D^{1}\right)} s_{t} \text { for each } D \subseteq C \backslash D^{1} \\
\sum_{i \in D} f_{i}\left(c, \omega^{2}\right) & =\sum_{t \in N_{g}(D) \backslash N_{g}\left(D^{1}\right)} s_{t} \text { for some } D \subseteq C \backslash D^{1}
\end{aligned}
$$

Then, there exists a unique largest set $D^{2}$ such that equation (4) holds. Observe that $\omega^{2} \geq \omega^{1}$. Since if $\omega^{2} \leq \omega^{1}$, we combine equations (2) and t4 to obtain 


$$
\sum_{c_{i} \in D^{1} \cup D^{2}} q_{i}\left(c, \omega^{1}\right) \geq \sum_{c_{i} \in D^{1}} q_{i}\left(c, \omega^{1}\right)+\sum_{c_{i} \in D^{2}} q_{i}\left(c, \omega^{2}\right)=\sum_{s_{t} \in N_{g}\left(D^{1} \cup D^{2}\right)} s_{t}
$$

which contradicts the choice of $D^{1}$ as the largest set satisfying equation (2).

Theorem 1. For each fairness principle $f$, the allocation rule $\varphi^{f}$ obtained by the ascending algorithm is pairwise $f$-robust.

Proof. Let $(s, c, g)$ be an allocation problem, $\varphi^{f}(s, c, g)=q$, and $i, j \in C$. First, suppose that the ascending algorithm assigns to $i$ and $j$ their allocations in the same iteration step, which means $q_{i}=f_{i}(c, \omega)$ and $q_{j}=f_{j}(c, \omega)$ for $\omega>0$. Consider the simple allocation problem $\left(\left(c_{i}, c_{j}\right), q_{i}+q_{j}\right)$. Since $f$ is consistent, $f\left(\left(c_{i}, c_{j}\right), q_{i}+q_{j}\right)=$ $\left(q_{i}, q_{j}\right)$. Hence, pairwise $f$-robustness is trivially satisfied.

Now, suppose that the ascending algorithm assigns to $i$ and $j$ their allocations in different iteration steps, meanings $q_{i}=f_{i}(c, \omega)$ and $q_{j}=f_{j}\left(c, \omega^{\prime}\right)$ for $\omega, \omega^{\prime}>0$, which are the parameters obtained from the ascending algorithm. Assume without loss of generality that $\omega^{\prime}>\omega$. By resource monotonicity, we have $q_{i}=f_{i}(c, \omega) \leq$ $f_{i}\left(c, \omega^{\prime}\right)$ and $q_{j}=f_{j}\left(c, \omega^{\prime}\right) \geq f_{j}(c, \omega)$. If one of these inequalities is not strict, then $f\left(\left(c_{i}, c_{j}\right), q_{i}+q_{j}\right)=\left(q_{i}, q_{j}\right)$ by the same argument presented above. Hence, assume that $q_{i}=f_{i}(c, \omega)<f_{i}\left(c, \omega^{\prime}\right)$ and $q_{j}=f_{j}\left(c, \omega^{\prime}\right)>f_{j}(c, \omega)$. Let $f_{i}\left(c, \omega^{\prime}\right)=\bar{q}_{i}$ and $f_{j}(c, \omega)=\overline{q_{j}}$. By consistency, we have $f\left(\left(c_{i}, c_{j}\right), q_{i}+\bar{q}_{j}\right)=\left(q_{i}, \overline{q_{j}}\right)$ and $f\left(\left(c_{i}, c_{j}\right), \bar{q}_{i}+\right.$ $\left.q_{j}\right)=\left(\bar{q}_{i}, q_{j}\right)$. Then, by resource monotonicity, we have $f_{i}\left(\left(c_{i}, c_{j}\right), q_{i}+q_{j}\right) \geq q_{i}$ and $f_{j}\left(\left(c_{i}, c_{j}\right), q_{i}+q_{j}\right) \leq q_{i}$. If one of these inequalities are weak, then pairwise $f$ robustness is satisfied. Hence, assume that both are strict. Since the algorithm assigns $j$ 's allocation after $i$ 's allocation, there is no feasible allocation $q^{\prime}$ such that for each $k \neq i, j, q_{k}=q_{k}^{\prime}$ and $q_{i}^{\prime}>q_{i}$. The reason is that each citizen who receives his allocation at some step $h$ obtains no resource from the sources, he shares with citizens who receive their allocations in the steps earlier than $h$. Hence, $\varphi^{f}$ obtained by the ascending algorithm is pairwise $f$-robust.

Theorem 2. For each fairness principle $f$, there exists a unique allocation rule $\varphi^{f}$ which is pairwise $f$-robust.

Proof. Let $\varphi^{f}$ be the allocation rule given by the ascending algorithm. Suppose there exists some other rule $\varphi \neq \varphi^{f}$ which also satisfies pairwise $f$-robustness. Then, there exists an allocation problem $(s, c, g)$ such that $\varphi(s, c, g)=q^{\prime} \neq q=\varphi^{f}(s, c, g)$.

Let $D^{1}, D^{2}, \ldots, D^{h}$ be the set of citizens which are allocated in steps $1,2, \ldots, h$ of the ascending algorithm, respectively. Suppose that there exists $i \in D^{1}$ such 
that $q_{i}^{\prime}<q_{i}$. Then, $q_{i}=f_{i}(c, \omega)$ for some $\omega>0$. Let $\phi^{\prime}$ be a flow which supports the allocation $q^{\prime}$. Consider the set of sources $S^{1}=N_{g}(i)$ connected to $i$. Let $C^{1}=\left\{j \in C: \exists t \in S^{1}\right.$ such that $\left.\phi_{t j}^{\prime}>0\right\}$. This set is non-empty, because the resources which were going to $i$ in $q$, now must be allocated to other citizens which share sources with $i$, due to the efficiency of the allocation $q^{\prime}$.

If there exists $j \in C^{1}$ such that $q_{j}^{\prime}>f_{j}(c, \omega)$, then it is possible to transfer some positive amount from $j$ to $i$ through the path $j t, t i$ without changing the allocations of citizens other than $i$ and $j$, contradicting pairwise $f$-robustness.

So, suppose that for each citizen $j \in C^{1}, q_{j}^{\prime} \leq f_{j}(c, \omega)$. Consider the set $S^{2}=N_{g}\left(C^{1}\right)$ and $C^{2}=\left\{j \in C: \exists t \in S^{2}\right.$ such that $\left.\phi_{t j}^{\prime}>0\right\}$. Since $q_{i}^{\prime}<q_{i}$ and for each citizen $j \in C^{1}, q_{j}^{\prime} \leq f_{j}(c, \omega), C^{2}$ is non-empty. If there exists $j \in C^{2}$ such that $q_{j}^{\prime}>f_{j}(c, \omega)$, then it is possible to transfer some positive amount from $j$ to $i$ through a path $j_{2} t_{2}, t_{2} j_{1}, j_{1} t_{1}, t_{1} i$ for some $j_{2} \in C^{2}, t_{2} \in S^{2}, j_{1} \in C^{1}$, and $t_{1} \in S^{1}$, without changing the allocations of citizens other than $i$ and $j_{2}$, contradicting pairwise $f$-robustness.

If there exists no $j \in C^{2}$ such that $q_{j}^{\prime}>f_{j}(c, \omega)$, then we continue iteratively to look for a $j$ such that $q_{j}^{\prime}>q_{j}$. Such a $j$ exists, because $q_{i}^{\prime}<q_{i}$ and the resource allocation rule $\varphi$ is efficient. Then, it is possible to make a transfer from $j$ to $i$ without changing the allocations of other citizens.

If for each $i \in D^{1}, q_{i}^{\prime} \geq q_{i}$, then we have $q_{i}^{\prime}=q_{i}$ for all $i \in D^{1}$. Since $q \neq q^{\prime}$, there exists $i \in D^{h_{1}}$ such that for each $h_{2}<h_{1}$ and each $j \in D^{h_{2}}, q_{j}^{\prime}=q_{j}$ and we can apply the same iterative argument starting from $i$ to find a contradiction to pairwise $f$-robustness.

Hence, $\varphi^{f}$ is the unique allocation rule which is pairwise $f$-robust.

\section{Three allocation rules}

\subsection{Egalitarian Allocation Rule}

The first fairness principle we extend is egalitarianism.

Egalitarian Rule, $\boldsymbol{e}$ : For each $P=(c, \omega) \in \mathcal{P}$, egalitarian rule assigns an allocation $e(c, \omega)=q$ such that for each $i \in C, q_{i}=\min \left\{c_{i}, \lambda\right\}$ where $\lambda$ solves $\sum_{i \in C} \min \left\{c_{i}, \lambda\right\}=\omega$.

We extend egalitarianism to obtain the egalitarian allocation rule $\varphi^{e}(s, c, g)=$ $q$. Instead of the extension algorithm proposed in the previous section, we use an 
equivalent, but more revealing ascending algorithm based on the following system $E(\lambda)$ of inequalities where $\lambda$ is a non-negative parameter $3^{3}$

$$
\sum_{j \in D} \lambda \wedge c_{j} \leq \sum_{t \in N_{g}(D)} s_{t} \text { for all } D \subseteq C
$$

For $\lambda=0$, equation (6) is satisfied for each $D \subseteq C$. For $\lambda=\infty$, there exists $D \subseteq C$ such that

$$
\sum_{j \in D} \lambda \wedge c_{j}>\sum_{t \in N_{g}(D)} s_{t}
$$

by construction. Hence, there exists a lowest $\lambda^{1}$, strictly positive, such that

$$
\sum_{j \in D} \lambda^{1} \wedge c_{j}=\sum_{t \in N_{g}(D)} s_{t} \text { for some } D \subseteq C
$$

As $\sum_{t \in N_{g}(D)} s_{t}$ is a submodular function of $D$, there exists a unique largest $D^{1}$ such that equation (7) holds for. The egalitarian allocation $\varphi^{e}(s, c, g)=q$ is obtained by setting

$$
q_{j}=\lambda^{1} \wedge c_{j} \text { for } j \in D^{1}
$$

and assigning other agents their egalitarian allocation in the reduced problem $(s \backslash$ $\left.N_{g}\left(D^{1}\right), c \backslash D^{1}, g \backslash\left(N_{g}\left(D^{1}\right) \cup D^{1}\right)\right)$. That is, we look for the lowest $\lambda^{2}>0$ such that

$$
\sum_{j \in D} \lambda^{2} \wedge c_{j}=\sum_{t \in N_{g}(D) \backslash N_{g}\left(D^{1}\right)} s_{t} \text { for some } D \subseteq C \backslash D^{1}
$$

There exists a unique largest set $D^{2}$ such that equation (8) holds for. If $\lambda^{2} \leq \lambda^{1}$, we combine equations (7) and (8) to obtain

$$
\sum_{j \in D^{1} \cup D^{2}} \lambda^{1} \wedge c_{j} \geq \sum_{j \in D^{1}} \lambda^{1} \wedge c_{j}+\sum_{j \in D^{2}} \lambda^{2} \wedge c_{j}=\sum_{t \in N_{g}\left(D^{1} \cup D^{2}\right)} s_{t}
$$

which contradicts the choice of $D^{1}$ as the largest set satisfying equation (7).

The egalitarian allocation rule is $\varphi^{e}(s, c, g)=q$ obtained in the algorithm above. For an example of the egalitarian allocation rule, see Figure 2.

\footnotetext{
${ }^{3}$ Note that for each $\lambda \in \mathbb{R}_{+}$, each $c \in \mathbb{R}_{+}^{n}$, and each $j=1,2, \ldots, n, \lambda \wedge c_{j} \equiv \min \left\{\lambda, c_{j}\right\}$.
} 


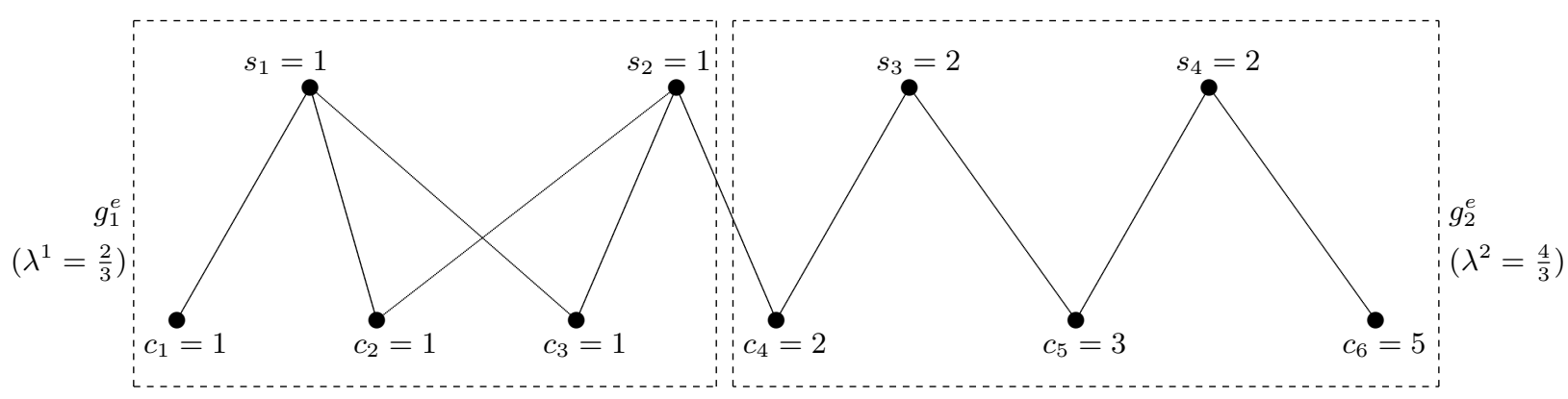

Figure 2: Egalitarian allocation rule: For the allocation problem $R=(s, c, g)$, the egalitarian allocation is $\varphi^{e}(s, c, g)=\left(\frac{2}{3}, \frac{2}{3}, \frac{2}{3}, \frac{4}{3}, \frac{4}{3}, \frac{4}{3}\right)$. In the algorithm, the lowest $\lambda^{1}$ satisfying equation $(7)$ is $\frac{2}{3}$ and the largest set satisfying equation 77 is $D^{1}=\{1,2,3\}$. Then, the lowest $\lambda^{2}$ satisfying equation $(8)$ is $\frac{4}{3}$ and the largest set satisfying equation 8 is $D^{2}=\{4,5,6\}$.

\subsection{Proportional Allocation Rule}

The second fairness principle is proportionality.

Proportional Rule, $\boldsymbol{p}$ : For each $P=(c, \omega) \in \mathcal{P}$, proportional rule assigns an allocation $\boldsymbol{p}(\boldsymbol{c}, \boldsymbol{\omega})=q=\pi c$ where $\pi=\frac{\omega}{\sum_{i \in C} c_{i}}$.

We obtain the proportional allocation rule $\varphi^{p}(s, c, g)=q$ by an ascending algorithm based on the following system $P(\pi)$ of inequalities where $\pi$ is a nonnegative parameter.

$$
\sum_{i \in D} \pi \cdot c_{i} \leq \sum_{t \in N_{g}(D)} s_{t} \text { for all } D \subseteq C
$$

For $\pi=0$, equation $(9)$ is satisfied for all $D \subseteq C$. For $\pi=1$ there exists $D \subseteq C$ such that

$$
\sum_{i \in D} \pi \cdot c_{i}>\sum_{t \in N_{g}(D)} s_{t}
$$

by construction. Hence, there exists a lowest $\pi^{1}$, strictly positive, such that

$$
\sum_{i \in D} \pi^{1} \cdot c_{i}=\sum_{t \in N_{g}(D)} s_{t} \text { for some } D \subseteq C
$$


As $\sum_{t \in N_{g}(D)} s_{t}$ is a submodular function of $D$, there exists a unique largest $D^{1}$ such that equation 10 holds for. The proportional allocation rule $\varphi^{p}(s, c, g)=q$ is obtained by setting

$$
q_{i}=\pi^{1} . c_{i} \text { for } i \in D^{1}
$$

and assigning other agents their constrained proportional allocation in the reduced problem $\left(s \backslash N_{g}\left(D^{1}\right), c \backslash D^{1}, g \backslash\left(N_{g}\left(D^{1}\right) \cup D^{1}\right)\right)$. That is, we look for the lowest $\pi^{2}>0$ such that

$$
\sum_{i \in D} \pi^{2} \cdot c_{i}=\sum_{t \in N_{g}(D) \backslash N_{g}\left(D^{1}\right)} s_{t} \text { for some } D \subseteq C \backslash D^{1}
$$

There exists a unique largest set $D^{2}$ such that equation 11 holds for. If $\pi^{2} \leq \pi^{1}$, we combine equations 10 and 11 to obtain

$$
\sum_{i \in D^{1} \cup D^{2}} \pi^{1} \cdot c_{i} \geq \sum_{i \in D^{1}} \pi^{1} \cdot c_{i}+\sum_{i \in D^{2}} \pi^{2} \cdot c_{i}=\sum_{t \in N_{g}\left(D^{1} \cup D^{2}\right)} s_{t}
$$

which contradicts the choice of $D^{1}$ as the largest set satisfying equation 10 .

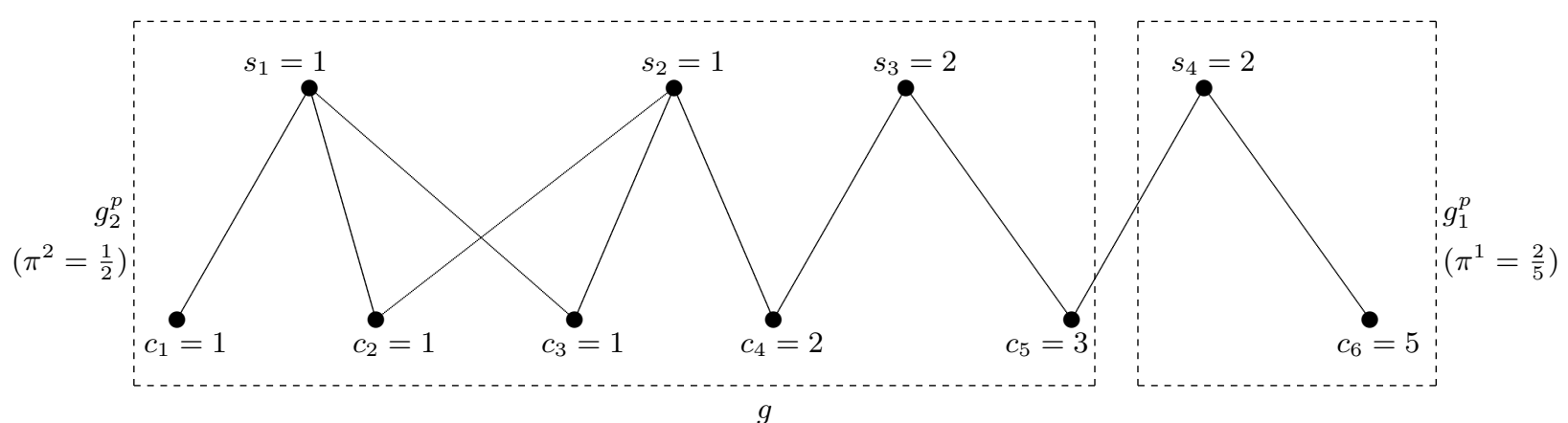

Figure 3: Proportional allocation rule: For the allocation problem $R=$ $(s, c, g)$, the proportional allocation is $\varphi^{p}(s, c, g)=\left(\frac{1}{2}, \frac{1}{2}, \frac{1}{2}, 1, \frac{3}{2}, 2\right)$. In the algorithm, the lowest $\pi^{1}$ satisfying equation 10 is $\frac{1}{2}$ and the largest set satisfying equation 10 is $D^{1}=\{1,2,3,4,5\}$. Then, the lowest $\pi^{2}$ satisfying equation 11 is $\frac{2}{5}$ and the largest set satisfying equation 11 is $D^{2}=\{6\}$.

The proportional allocation rule is $\varphi^{p}(s, \boldsymbol{c}, \boldsymbol{g})=\boldsymbol{q}$ obtained in the algorithm above. For an example of the proportional allocation rule, see Figure 3. 


\subsection{Equal Sacrifice Allocation Rule}

The third fairness principle is egalitarianism in terms of the sacrifices required from the agents.

Equal Sacrifice Rule, $\boldsymbol{l}$ : For each $P=(c, \omega) \in \mathcal{P}$, equal sacrifice rule assigns an allocation $\boldsymbol{l}(\boldsymbol{c}, \boldsymbol{\omega})=q$ such that for each $i \in C, q_{i}=\max \left\{0, c_{i}-\sigma\right\}$ where $\sigma$ solves $\sum_{i \in C} \max \left\{0, c_{i}-\sigma\right\}=\omega$.

We obtain the equal sacrifice allocation rule $\varphi^{l}(s, c, g)=q$ by a descending algorithm based on the following system $S(\sigma)$ of inequalities where $\sigma$ is a nonnegative parameter $4^{4}$

$$
\sum_{i \in D} 0 \vee\left(c_{i}-\sigma\right) \leq \sum_{t \in N_{g}(D)} s_{t} \text { for all } D \subseteq C
$$

For $\sigma=\infty$, equation 12 is satisfied for all $D \subseteq C$. For $\sigma=0$ there exists $D \subseteq C$ such that

$$
\sum_{i \in D} 0 \vee\left(c_{i}-\sigma\right)>\sum_{t \in N_{g}(D)} s_{t}
$$

by construction. Hence, there exists a largest $\sigma^{1}$ such that

$$
\sum_{i \in D} 0 \vee\left(c_{i}-\sigma^{1}\right)=\sum_{t \in N_{g}(D)} s_{t} \text { for some } D \subseteq C
$$

As $\sum_{t \in N_{g}(D)} s_{t}$ is a submodular function of $D$, there exists a unique largest $D^{1}$ such that equation 13 holds for. The equal sacrifice allocation $\varphi^{l}(s, c, g)=q$ is obtained by setting

$$
q_{i}=0 \vee\left(c_{i}-\sigma^{1}\right) \text { for } i \in D^{1}
$$

and assigning other agents their equal sacrifice allocation in the reduced problem $\left(s \backslash N_{g}\left(D^{1}\right), c \backslash D^{1}, g \backslash\left(N_{g}\left(D^{1}\right) \cup D^{1}\right)\right)$. That is, we look for the largest $\sigma^{2}>0$ such that

$$
\sum_{i \in D} 0 \vee\left(c_{i}-\sigma^{2}\right)=\sum_{t \in N_{g}(D) \backslash N_{g}\left(D^{1}\right)} s_{t} \text { for some } D \subseteq C \backslash D^{1}
$$

There exists a unique largest set $D^{2}$ such that equation 14 holds for. If $\sigma^{2} \geq \sigma^{1}$, we combine equations 13 and 14 to obtain

\footnotetext{
${ }^{4}$ Note that for each $c \in \mathbb{R}_{+}^{n}$ and each $j=1,2, \ldots, n, 0 \vee\left(c_{i}-\sigma\right) \equiv \max \left\{0, c_{j}-\sigma\right\}$.
} 


$$
\sum_{i \in D^{1} \cup D^{2}} 0 \vee\left(c_{i}-\sigma^{1}\right) \geq \sum_{i \in D^{1}} 0 \vee\left(c_{i}-\sigma^{1}\right)+\sum_{i \in D^{2}} 0 \vee\left(c_{i}-\sigma^{2}\right)=\sum_{t \in N_{g}\left(D^{1} \cup D^{2}\right)} s_{t}
$$

which contradicts the choice of $D^{1}$ as the largest set satisfying equation 13 .

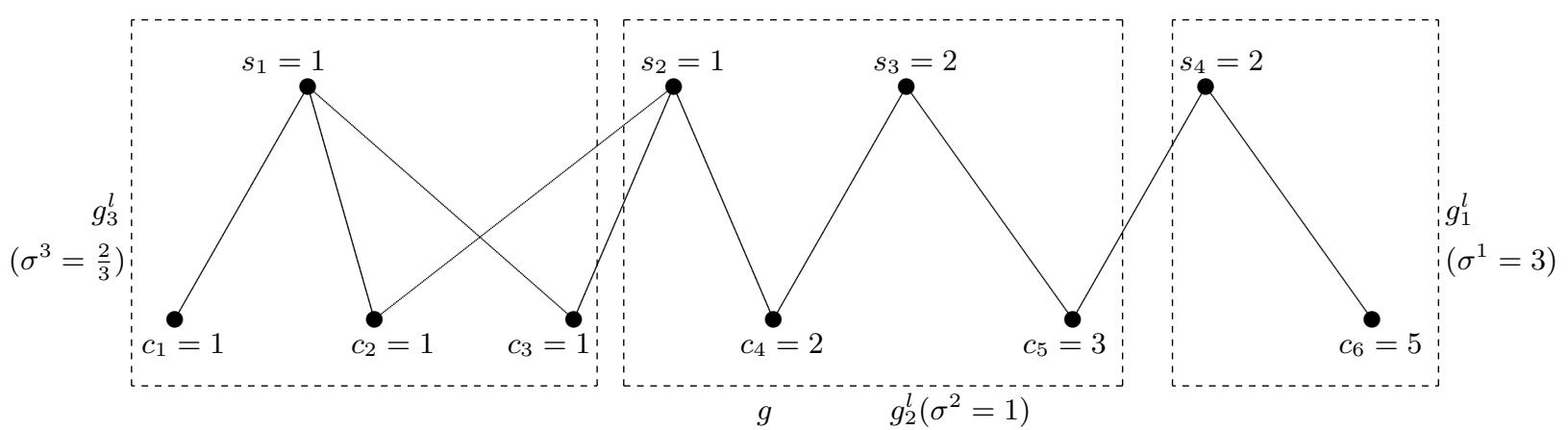

Figure 4: Equal sacrifice allocation rule: For the allocation problem $R=$ $(s, c, g)$, the equal sacrifice allocation is $\varphi^{l}(s, c, g)=\left(\frac{1}{3}, \frac{1}{3}, \frac{1}{3}, 1,2,2\right)$. In the algorithm, the highest $\sigma^{1}$ satisfying equation 13 is 3 and the largest set satisfying equation 13 is $D^{1}=\{6\}$. Then, the highest $\sigma^{2}$ satisfying equation 14 is 1 and the largest set satisfying equation 14 is $D^{2}=\{4,5\}$. Then, the highest $\sigma^{3}$ satisfying equation 14$\}^{k}$ ) (where $D^{1}$ is replaced with $D^{1} \cup D^{2}$ in equation (14) is $\frac{2}{3}$ and the largest set satisfying equation $14{ }^{*}$ ) is $D^{3}=\{1,2,3\}$.

The equal sacrifice allocation rule is $\varphi^{l}(s, c, g)=\boldsymbol{q}$ obtained in the algorithm above. For an example of the equal sacrifice allocation rule, see Figure 4.

\section{Conclusion}

Our results expand the scope of the existing literature on the claims problems. We provide a unique way to apply fairness principles to allocation problems on networks of sources and agents. Such problems with multiple sources are very commonly observed as exemplified in the introduction.

Moreover, the network model brings new theoretical questions. For example which axiomatic properties of simple rules carry over to allocation rules on networks? How can the axioms defined for simple allocations problems (e.g. no envy, 
composition up, consistency, etc.) can be extended to allocation rules on networks? The egalitarian rule for network allocation problems has been characterized with Pareto optimality, equal treatment of equals and strategy-proofness (Bochet et al. 2010). Is it possible to give a characterization of rules on how they respond to changes in the network structure? Similarly, the dual of an allocation problem (Thomson, 2006) is well defined when there is only one source. Is it possible to define the dual of an allocation problem on a network? In general, any question

which is relevant for simple allocation rules can now be asked for their extensions on networks as provided by our algorithm.

\section{References}

Ambec, S. and L. Ehlers (2008). Sharing a river among satiable agents. Games and Economic Behavior 64, 35-50.

Ambec, S. and Y. Sprumont (2002). Sharing a river. Journal of Economic Theory 107, 453-462.

Ansink, E. and H. P. Weikard (2009). Contested water rights. European Journal of Political Economy 25, 247-260.

Ansink, E. and H. P. Weikard (2011). A strategic model of social and economic networks. Social Choice and Welfare. (forthcoming).

Bjørndal, E. and K. Jörnsten (2010). Flow sharing and bankruptcy games. International Journal of Game Theory 39, 11-28.

Bochet, O., R. İlkılıç, and H. Moulin (2010). Egalitarianism under earmark constraints. mimeo. University of Bern, Bern, Switzerland.

Bochet, O., R. İlkılıç, H. Moulin, and J. Sethuraman (2011). Balancing supply and demand under bilateral constraints. Theoretical Economics. (forthcoming).

Branzei, R., G. Ferrari, V. Fragnelli, and S. Tijs (2008). A flow approach to bankruptcy problems. AUCO Czech Economic Review 2, 146-153.

Brown, J. (1979). The sharing problem. Operations Research 27, 324-340.

Hall, N. G. and R. Vohra (1993). Towards equitable distribution via proportional equity constraints. Mathematical Programming 58, 287-294. 
Hoekstra, A. (2006). The global dimension of water governance: Nine reasons for global arrangements in order to cope with local problems. Value of Water Research Report Series 20. UNESCO-IHE Institute for Water Education.

İlkılıç, R. (2007). Network of commons. mimeo. Maastricht University. Maastricht, the Netherlands.

Kar, A. and O. Kıbris (2008). Allocating multiple estates among agents with single-peaked preferences. Social Choice and Welfare 31, 641-666.

Klaus, B., H. Peters, and T. Storcken (1997). Reallocation of an infinitely divisible good. Economic Theory 10, 305-333.

Klaus, B., H. Peters, and T. Storcken (1998). Strategy-proof division with singlepeaked preferences and individual endowments. Social Choice and Welfare 15, $297-311$.

Megiddo, N. (1974). Optimal flows in networks with multiple sources and sinks. Mathematical Programming 7, 97-107.

Megiddo, N. (1977). A good algorithm for lexicographically optimal flows in multiterminal networks. Bulletin of the American Mathematical Society 83, 407-409.

Moulin, H. (1999). Rationing a commodity along fixed paths. Journal of Economic Theory $84,41-72$.

Moulin, H. and J. Sethuraman (2011). The bipartite rationing problem. mimeo. Rice University, Houston, TX, USA.

O'Neill, B. (1982). A problem of rights arbitration from the talmud. Mathematical Social Sciences 2, 345-371.

Özdamar, O., E. Ekinci, and B. Küçükyazıcı (2004). Emergency logistics planning in natural disasters. Annals of Operations Research 129, 217-245.

Sprumont, Y. (1991). The division problem with single-peaked preferences: A characterization of the uniform allocation rule. Econometrica 59, 509-519.

Thomson, W. (2003). Axiomatic analysis of bankruptcy and taxation problems: a survey. Mathematical Social Sciences 45, 249-297.

Thomson, W. (2006). How to divide when there isnt enough: From the talmud to game theory. mimeo. University of Rochester, Rochester, NY, USA. 